\title{
Problemas oculares relacionados ao uso de telas em pacientes pediátricos
}

\author{
Eye problems related to the use of screens in pediatric patients
}

Problemas oculares relacionados con el uso de pantallas en pacientes pediátricos

Milla Morena Persiano Schamache ${ }^{1 *}$, Lavínia Gonzaga Taveira1, João Vitor de Freitas Martins ${ }^{1}$, Carlos Vitor Mello Moreira1, Vitória Carolina Sampaio Bastos França1, Laura Lutterbach Dalbuone Pinheiro ${ }^{1}$, Thaiana Raad Drumond ${ }^{1}$, Diorransem Sales Gomes ${ }^{2}$, Fernanda Ferreira Arrabal', Vanessa Stella de Souza'.

\section{RESUMO}

Objetivo: Descrever os problemas visuais relacionados ao uso de telas por pacientes pediátricos e evidenciar quais são os fatores de risco modificáveis. Revisão bibliográfica: Atualmente, devido às aulas remotas, 36,9\% das crianças utilizam dispositivos digitais por mais de 5 horas por dia, antes da pandemia, tal tempo de uso ocorria em apenas $1,8 \%$ das crianças. É frequente a ocorrência de sintomas oculares como xeroftalmia, prurido, sensação de corpo estranho, lacrimejamento e visão turva em pacientes pediátricos que passam muito tempo diante de telas de computadores, tablets e celulares. Ademais, cabe ressaltar que existem evidências de que um maior tempo ao ar livre diminui a ocorrência de miopia. Infelizmente, estudos demonstram que quanto maior o tempo de exposição a telas, menor é o tempo de exposição ao ar livre. Considerações finais: Assim, deve haver conscientização da população a fim de diminuir o tempo de uso de dispositivos eletrônicos e aumentar o tempo ao ar livre, a fim de diminuir a frequência desses distúrbios.

Palavras-chave: Crianças, Visão, Smartphone.

\begin{abstract}
Objective: To describe the visual problems related to the use of screens by pediatric patients and highlight the modifiable risk factors. Bibliographic review: Currently, due to remote classes, $36.9 \%$ of children use digital devices for more than 5 hours a day, before the pandemic, such time of use occurred in only $1.8 \%$ of children. Eye symptoms such as xerophthalmia, pruritus, foreign body sensation, tearing and blurred vision often occur in pediatric patients who spend a lot of time in front of computer screens, tablets and cell phones. Furthermore, it is noteworthy that there is evidence that more time outdoors reduces the occurrence of myopia. Unfortunately, studies show that the longer the exposure time to screens, the shorter the exposure time outdoors. Final considerations: Thus, there must be awareness of the population in order to reduce the time of using electronic devices and increase the time outdoors, in order to reduce the frequency of these disturbances.
\end{abstract}

Keywords: Child, Vision, Smartphone.

\section{RESUMEN}

Objetivo: Describir los problemas visuales relacionados con el uso de pantallas por parte de pacientes pediátricos y destacar los factores de riesgo modificables. Revisión bibliográfica: Actualmente, debido a las clases a distancia, el $36,9 \%$ de los niños utilizan dispositivos digitales durante más de 5 horas al día, antes de la pandemia, este tiempo de uso se da en solo el 1,8\% de los niños. Los síntomas oculares como xeroftalmía, prurito, sensación de cuerpo extraño, lagrimeo y visión borrosa se presentan a menudo en pacientes pediátricos que pasan mucho tiempo frente a pantallas de computadora, tabletas y teléfonos celulares. Además, cabe destacar que existe evidencia de que pasar más tiempo al aire libre reduce la aparición de miopía. Desafortunadamente, los estudios muestran que cuanto mayor es el tiempo de exposición a las pantallas, menor es el tiempo de exposición al aire libre. Consideraciones finales: Por tanto, se debe sensibilizar a la población para reducir el tiempo de uso de dispositivos electrónicos y aumentar el tiempo al aire libre, con el fin de reducir la frecuencia de estas perturbaciones.

Palabras clave: Ninõ, Visión, Teléfono inteligente.

${ }^{1}$ Universidade Iguaçu (UNIG), Itaperuna - RJ. *E-mail: milla_persiano@hotmail.com

${ }^{2}$ Centro Universitário Redentor (UniREDENTOR), Itaperuna - RJ.

SUBMETIDO EM: 9/2021

ACEITO EM: 9/2021

PUBLICADO EM: 9/2021 


\section{INTRODUÇÃO}

O uso de dispositivos eletrônicos como celulares, tablets e computadores por crianças e adolescentes tem aumentado significativamente ao longo dos anos. Em 2018, cerca de 42\% das crianças com idade entre 5 e 7 anos em todo o mundo possuíam um tablet e $5 \%$ delas tinham seu próprio celular (OFFICE OF COMMUNICATIONS (OFCON), 2019). Vale salientar que a atual situação vivenciada pelo mundo devido à pandemia de COVID-19, causada pelo vírus SARS-CoV-2, contribui ainda mais com o aumento do tempo de uso de dispositivos eletrônicos (MOHAN A, et al., 2021).

O isolamento social foi necessário com o objetivo de diminuir a transmissão do vírus, consequentemente, as pessoas passaram a permanecer mais tempo dentro das suas residências. Além disso, as aulas presenciais nas escolas foram suspensas, fazendo com que os estudantes ficassem ainda mais dependentes dos dispositivos eletrônicos tanto para o aprendizado escolar quanto para a socialização com amigos e familiares. Também devido ao isolamento social, muitos indivíduos passaram a trabalhar das suas próprias casas. Dessa forma, as crianças e os adolescentes estão vendo os pais constantemente utilizando telas o que acaba estimulando e aumentando o interesse pelo uso de aparelhos eletrônicos (CHANG FC, et al., 2018).

O uso dessas tecnologias está associado a diversos benefícios tais como o desenvolvimento de raciocínio rápido, a aprendizagem precoce e a exposição a novas ideias e a novos conhecimentos (ALVAREZ PC, et al., 2020; LISSAK G, 2018; REID CHASSIAKOS YL, et al., 2016). Contudo, é válido ressaltar que tem sido observado que o uso excessivo de dispositivos eletrônicos está relacionado com diversas consequências sociais, psicológicas, físicas e neurológicas em crianças e adolescentes (LISSAK G, 2018; REID CHASSIAKOS YL, et al., 2016).

Ressalta-se ainda que a exposição excessiva a telas de computadores, celulares e tablets tem sido relacionada com comportamentos de imediatismo em crianças e adolescentes (ALVAREZ PC, et al., 2020; LISSAK G, 2018). Além disso, a internet pode ser um ambiente perigoso para as crianças, podendo ocorrer exposição a conteúdos inadequados para a idade e até mesmo inseguros, colocando em risco a segurança das crianças e dos adolescentes que fazem uso indiscriminado das mídias sociais (ALVAREZ PC, et al., 2020; LISSAK G, 2018; REID CHASSIAKOS YL, et al., 2016).

A Sociedade Brasileira de Pediatria alerta sobre os prejuízos à saúde, relacionados ao uso precoce, excessivo e prolongado de telas durante a infância (SOCIEDADE BRASILEIRA DE PEDIATRIA, 2019). São diversos os problemas médicos relacionados a era digital, sendo frequente os problemas de saúde mental como irritabilidade, ansiedade, depressão e transtornos de imagem corporal e da autoestima (ALVAREZ PC, et al., 2020; HOKBY S, et al., 2016; LISSAK G, 2018). A internet e redes sociais podem, portanto, ser extremamente prejudiciais para crianças e adolescentes se usada sem supervisão e por quantidade de tempo excessiva (ALVAREZ PC, et al., 2020; HOKBY S, et al., 2016; LISSAK G, 2018; REID CHASSIAKOS YL, et al., 2016).

Além disso, dentre as consequências causadas pelo uso excessivo de aparelhos eletrônicos e redes sociais, também foram descritos transtornos de déficit de atenção e hiperatividade, transtornos do sono, sobrepeso, obesidade, anorexia, bulimia, sedentarismo, transtornos posturais, problemas musculoesqueléticos e transtornos visuais. As redes sociais podem impactar de maneira severa a percepção de imagem corporal e ajudar no desenvolvimento de transtornos alimentares (ALVAREZ PC, et al., 2020; COPETTI AVS, QUIROGA CV, 2018; HOKBY S, et al., 2016; LISSAK G, 2018; REID CHASSIAKOS YL, et al., 2016).

Diante disso, a recomendação presente no manual de orientação publicado em 2019 pela Sociedade Brasileira de Pediatria é que o tempo de uso de telas por crianças seja limitado a 1 hora por dia entre 2 e 5 anos, 1 a 2 horas por dia entre 6 e 10 anos e 2 a 3 horas por dia entre 11 e 18 anos. Em relação a crianças menores que 2 anos, a recomendação é evitar a exposição às telas, mesmo que seja feita de forma passiva (SOCIEDADE BRASILEIRA DE PEDIATRIA, 2019). Entretanto, essa não é a realidade de grande parte das 
crianças e adolescentes no mundo. Atualmente, a maioria delas utilizam dispositivos eletrônicos muitas horas por dia, tendo o uso aumentado ainda mais com a ocorrência de aulas online durante a pandemia de COVID19 (MOHAN A, et al., 2021).

Cabe ressaltar que diversos problemas visuais relacionados ao uso de dispositivos como celulares, tablets, computadores e videogames já foram descritos na literatura. Já se sabe que os distúrbios da visão podem causar muitas consequências nos indivíduos acometidos, como prejuízo no desempenho escolar, cefaleia e diminuição na qualidade de vida (LISSAK G, 2018; REID CHASSIAKOS YL, et al., 2016).

Dessa forma, torna-se crucial a compreensão sobre tais distúrbios e a identificação do que pode ser feito a fim de minimizá-los. Sendo assim, o objetivo desta revisão narrativa foi descrever os problemas visuais relacionados ao uso de telas por pacientes pediátricos, bem como evidenciar quais são os seus fatores de risco modificáveis.

\section{REVISÃO BIBLIOGRÁFICA}

O uso excessivo de telas por crianças e por adolescentes é frequentemente relatado pelos pais durante a realização da consulta pediátrica. Destaca-se que o tempo de uso de dispositivos eletrônicos por crianças e adolescentes está ainda maior atualmente. Isso se deve ao fato da pandemia de COVID-19 ter impactado de maneira significativa as escolas que visando uma diminuição da transmissão do vírus tiveram suas aulas presenciais suspensas e adaptaram o modelo de ensino para continuação das aulas (MOHAN A, et al., 2021).

A fim de diminuir as consequências causadas pela paralização das aulas, grande parte das escolas optaram por substituir o modelo tradicional de ensino pelo ensino a distância. Neste novo modelo, as aulas são ministradas pelos professores de maneira remota e são assistidas pelos alunos através de dispositivos eletrônicos. Embora o ensino a distância tenha possibilitado a continuidade do aprendizado dos estudantes, esse modelo aumentou ainda mais o tempo em que crianças e adolescentes ficam expostos a telas de celulares e computadores. Atualmente, devido às aulas remotas, $36,9 \%$ das crianças utilizam dispositivos digitais por mais de 5 horas por dia, antes da pandemia, tal tempo de uso ocorria em apenas 1,8\% das crianças (MOHAN A, et al., 2021).

Dentre as consequências do uso excessivo desse tipo de tecnologia está a maior ocorrência de problemas visuais, tais como miopia e xeroftalmia em crianças e adolescentes que passam muito tempo utilizando dispositivos eletrônicos como celulares, tablets e computadores (MOHAN A, et al., 2021; KIM J, et al., 2016). A miopia é a condição oftalmológica mais comum no mundo, sendo considerada um problema mundial de saúde pública. É importante evidenciar que o aumento da sua prevalência resulta em um importante impacto socioeconômico, gerando custos tanto com as consultas oftalmológicas como com a compra de óculos e lentes corretoras (RAMAMURTHY D, et al., 2015; CHUA J, WONG TY., 2016). Sendo assim, torna-se imprescindível a identificação dos fatores de risco modificáveis relacionados à miopia para, dessa forma, promover políticas públicas visando à diminuição da sua ocorrência (MOHAN A, et al., 2021; KIM J, et al., 2016).

É importante destacar que existem fatores de risco para miopia que podem ser evitáveis. Um desses fatores é o uso de aparelhos eletrônicos como celulares, tablets e computadores por tempo excessivo por crianças e adolescentes. Diversos estudos demonstram que existe relação entre o uso prolongado de aparelhos eletrônicos e o desenvolvimento de miopia. Infelizmente, nos últimos 3 anos, o tempo médio de uso de dispositivos eletrônicos por crianças entre 8 e 12 anos tem aumentado 49 minutos por dia, com um tempo médio por dia de 4 horas e 18 minutos em 2016 e de 5 horas e 7 minutos em 2019. Percebe-se também que crianças mais velhas costumam passar um maior tempo diante de telas de dispositivos eletrônicos (ALVAREZ PC, et al., 2020).

A desfocagem hiperópica durante a visualização de dispositivos eletrônicos portáteis é observada com maior frequência em crianças não míopes do que em crianças míopes (SAH RP, et al., 2020). Além disso, foi notado um aumento na prevalência de miopia em crianças espanholas com idade entre 5 e 7 anos, sendo necessário destacar que cerca de $43,3 \%$ dessas crianças ficaram mais de 3 horas por dia em atividades de proximidade e apenas $9,7 \%$ ficaram mais de 2,5 horas por dia expostas ao ar livre durante o período em que 
se realizou o estudo (ALVAREZ-PEREGRINA CC, et al., 2019). Dessa forma, medidas devem ser tomadas a fim de diminuir o tempo diário em que crianças e adolescentes são expostas a telas. É importante que sejam desenvolvidas políticas públicas visando à conscientização dos pais acerca dos riscos causados pelo uso excessivo de dispositivos digitais (ALVAREZ-PEREGRINA CC, et al., 2019).

Os computadores são muito utilizados por todas as idades e estima-se que aproximadamente $50 \%$ dos usuários de computador apresentam a síndrome da visão computacional. Essa síndrome está relacionada com uma ampla gama de sintomas oculares e visuais vinculados ao estresse da visão acomodativa e a ocorrência de olho seco (SHEPPARD AL, WOLFFSOHN JS, 2018). Alguns pesquisadores defendem a ideia de que o uso de óculos com lentes com filtro de luz azul ajuda a evitar a ocorrência da síndrome, diminuindo assim os sintomas visuais relacionados ao uso de computadores. Em um estudo transversal realizado para avaliar, através de um questionário, de qual maneira os dispositivos digitais afetam a visão binocular e acomodativa de pré-adolescentes, foi observado que os indivíduos fortemente acometidos com a síndrome da visão computacional desenvolveram mais distúrbios de vergência (DE-HITA-CANTALEJO C, et al., 2021).

Uma pesquisa observacional foi realizada com o objetivo de examinar uma possível relação entre o tempo jogando videogames e a ocorrência de problemas visuais em crianças com idade entre 3 e 10 anos. Tal estudo evidenciou que existe uma associação entre o tempo jogando videogames com sintomas visuais que podem fazer parte de uma síndrome de visão de videogame ainda não definida. É importante destacar, contudo, que mais estudos devem ser realizados a fim de identificar possíveis relações causais entre 0 videogame e problemas visuais em crianças e adolescentes (RECHICHI C, et al., 2017).

Em tal estudo, as crianças que costumavam passar mais de 30 minutos por dia jogando videogames e que jogavam todos os dias apresentaram cefaleia, tique palpebral, diplopia transitória, tontura e erros de refração com mais frequência do que as crianças que jogavam videogame por menos de 30 minutos por dia e não todos os dias. Assim, percebe-se que os pais devem ficar atentos ao tempo que seus filhos passam jogando videogames, devendo haver uma boa comunicação entre pais e filho. Assim, tal atividade de lazer se torna menos prejudicial à saúde da criança. Ademais, cabe ressaltar que os familiares devem garantir que os jogos comprados sejam próprios para a idade das crianças e adolescentes (RECHICHI C, et al., 2017).

É frequente a ocorrência de sintomas oculares em crianças e adolescentes que passam muito tempo diante de telas de dispositivos eletrônicos. Assim, pacientes pediátricos podem vir a ter sintomas como xeroftalmia, prurido, sensação de corpo estranho, lacrimejamento e visão turva devido ao uso excessivo de telas (MOHAN A, et al., 2021; KIM J, et al., 2016).

Uma pesquisa recente evidenciou que esses sintomas são frequentes em $50,23 \%$ das crianças que estão tendo aulas a distância devido à pandemia de COVID-19. Além disso, em relação à gravidade dos sintomas, $26,3 \%$ dos participantes tinham sintomas leves, 12,9\% tinham sintomas moderados e $11,1 \%$ tinham sintomas graves. Além disso, cerca de $50 \%$ dos pais relataram que perceberam uma piora na visão dos filhos depois da implementação das aulas online (MOHAN A, et al., 2021). Infelizmente, enquanto a pandemia de COVID19 não permitir o retorno total das aulas presenciais, será grande o número de crianças e adolescentes que utilizam dispositivos eletrônicos por mais tempo do que o recomendado pela Sociedade Brasileira de Pediatria. Dessa maneira, o uso fora das aulas deve ser ainda mais desestimulado a fim de evitar ficar muito tempo exposto a telas (MOHAN A, et al., 2021; KIM J, et al., 2016).

Um outro estudo, realizado em Cingapura, encontrou associação entre o uso de dispositivos móveis de toque e a ocorrência de sintomas musculoesqueléticos no pescoço, ombro, braços e costas. Contudo, nesta pesquisa, o uso de celulares e tablets não foi associado a ocorrência de sintomas visuais, ao uso de óculos ou à miopia. Vale destacar que por se tratar de questionários digitais mais estudos controlados devem ser realizados para confirmar tais achados (TOH SH, et al., 2020). Grande parte dos usuários de dispositivos eletrônicos realizam flexão defeituosa de suas costas e pescoço quando fazem uso destes dispositivos, sendo frequente, portanto, os sintomas musculoesqueléticos (FARES J, et al., 2017).

A ocorrência de olho seco, também conhecido como xeroftalmia, em pacientes pediátricos ocorre com maior frequência em usuários de dispositivos digitais que ficam muito tempo expostos a tela (BUABBAS AJ, 
et al., 2020; MOON JH, et al., 2014; MOON JH, et al., 2016; SHEPPARD AL, WOLFFSOHN JS, 2018). Quando se avalia os fatores de risco para ocorrência de olhos secos em escolares, pode-se perceber que o uso de celulares é um importante fator de risco para a ocorrência de olhos secos em crianças e adolescentes (MOON JH, et al., 2014).

$\mathrm{Na}$ Coreia, entre $80 \%$ e $90 \%$ das crianças e adolescentes utilizam celulares, observou-se que a frequência da doença do olho seco é maior entre as que residem em áreas urbanas, visto que $8,3 \%$ delas foram diagnosticadas com o distúrbio, enquanto apenas $2,8 \%$ das crianças que moravam em área rural receberam tal diagnóstico. Ademais, a taxa de uso de celulares foi de $61,3 \%$ no grupo que residia em áreas urbanas e de $51 \%$ no que residia em áreas rurais. Nota-se que o uso de celulares é mais frequente nas séries mais avançadas. Além disso, a ocorrência de olho seco é mais comum no grupo de crianças que utilizava os celulares por mais tempo, tal grupo também passou um menor tempo ao ar livre (MOON J, et al., 2016).

Diante dessas informações, pode-se entender que as crianças e adolescentes que moram em cidades estão sendo mais expostas à tecnologia e correm mais riscos de desenvolver problemas de saúde relacionados ao uso de aparelhos eletrônicos (MOON JH, et al., 2016).

É importante destacar que outro fator de risco modificável é a realização de atividades ao ar livre (RAMAMURTHY D, et al., 2015). Existem diversas evidências recentes de que um tempo maior realizando atividades ao ar livre tem relação com a diminuição da ocorrência de problemas oculares como miopia em crianças e adolescentes (ALVAREZ PC, et al., 2020; ALVAREZ-PEREGRINA CC, et al., 2019; RAMAMURTHY D, et al., 2015; THEOPHANUS C, et al., 2018). As pesquisas evidenciam que este é um fator ambiental modificável que protege os indivíduos contra a miopia e tal efeito protetor possivelmente tem relação com a alta intensidade da luz ao ar livre, com a cromaticidade da luz do dia ou com o aumento dos níveis de vitamina D (RAMAMURTHY D, et al., 2015).

Dessa forma, foi observado que a prevalência de miopia diminui à medida que se aumenta o tempo ao ar livre e que se aumenta o período de exposição à luz ultravioleta (ALVAREZ PC, et al., 2020). Destaca-se também que quanto mais velhas são as crianças, menor é o número de horas que elas ficam expostas ao ar livre (ALVAREZ PC, et al., 2020). Sendo assim, a prática de esportes pode ser uma ótima aliada não só na diminuição dos problemas cardiovasculares, da obesidade e do sedentarismo como também na diminuição do tempo de uso de celulares, computadores e tablets. Além disso, a prática de esportes ao ar livre resulta em um maior tempo de exposição aos raios ultravioletas, contribuindo assim para a diminuição da prevalência de miopia em crianças (BUABBAS AJ, et al., 2020).

Em 2019, a Organização Mundial de Saúde (OMS), publicou um relatório sobre a visão no qual se evidenciava o aumento na prevalência mundial da miopia, destacando que os casos de miopia devem aumentar de 1,95 bilhão de pessoas acometidas em 2010 para 3,36 bilhões em 2030. Foi evidenciado que este aumento tem relação com a redução do tempo passado ao ar livre, juntamente com o aumento do tempo realizando atividades que exigem visão para perto como ler, estudar, utilizar celular, computadores, dentre outros (OMS, 2019). Ressalta-se que em relação ao tempo ao ar livre, a Sociedade Brasileira de Pediatria recomenda que crianças fiquem ao menos 1 hora por dia em atividades em contato com a natureza, sendo este contato com a natureza um ótimo aliado para se diminuir a intoxicação digital vivenciada nos dias de hoje (SOCIEDADE BRASILEIRA DE PEDIATRIA, 2019).

Em relação a periodicidade das consultas com oftalmologistas a Sociedade Brasileira de Oftalmologia Pediátrica publicou a Diretriz Brasileira Acerca da Periodicidade do Exame Oftalmológico nas Crianças Saudáveis na primeira infância a fim de orientar melhor os profissionais de saúde. Essa Diretriz evidencia que um exame oftalmológico completo deve ser realizado por um oftalmologista em crianças com idade entre $3 \mathrm{e}$ 5 anos, preferencialmente aos 3 anos. Neste exame deve-se incluir a inspeção dos olhos e dos seus anexos, a avaliação da acuidade visual, utilizando optótipos adequados à idade, a avaliação da motilidade e o alinhamento ocular através de testes de cobertura simples e alternada, a refração cicloplegiada e a avaliação do fundo de olho dilatado. Caso os resultados obtidos sejam insatisfatórios ou inconclusivos o exame deverá ser repetido após 6 meses (ROSSETO JD, et al., 2020). 


\section{CONSIDERAÇÕES FINAIS}

Diante disso, nota-se que o uso de telas por pacientes pediátricos tem relação com a ocorrência de prurido ocular, xeroftalmia, sensação de corpo estranho, lacrimejamento, visão turva, miopia, tique palpebral e diplopia transitória. Ademais, é evidente que o tempo de exposição a telas por crianças e adolescentes está muito acima do tempo recomendado pela Sociedade Brasileira de Pediatria e pela Sociedade Brasileira de Oftalmologia Pediátrica. Destaca-se que existem fatores de risco para problemas oculares em crianças e adolescentes que podem ser evitados, como o tempo de uso de dispositivos eletrônicos e o tempo de exposição ao ar livre. Assim, deve haver conscientização da população a fim de diminuir o tempo de uso de dispositivos eletrônicos e aumentar o tempo ao ar livre, a fim de diminuir a frequência desses distúrbios.

\section{REFERÊNCIAS}

1. ALVAREZ-PEREGRINA CC, et al. Prevalence and Risk Factors of Myopia in Spain. Journal of ophthalmology, 2019; 2019: 3419576.

2. ALVAREZ-PEREGRINA CC, et al. The Relationship Between Screen and Outdoor Time With Rates of Myopia in Spanish Children. Frontiers in public health, 2020; 14(8): 560378.

3. BUABBAS AJ, et al. The detrimental impacts of smart technology device overuse among school students in Kuwait: a crosssectional survey. BMC pediatrics, 2020; 20(1): 524.

4. CHANG FC, et al. Computer/Mobile Device Screen Time of Children and Their Eye Care Behavior: The Roles of Risk Perception and Parenting. Cyberpsychology, behavior and social networking, 2018; 21(3): 179-186.

5. CHUA J, WONG TY. Myopia-The Silent Epidemic That Should Not Be Ignored. JAMA Ophthalmology, 2016; 134(12): 13631364.

6. COPETTI AVS, QUIROGA CV. A influência da mídia nos transtornos alimentares e na autoimagem em adolescentes. Rev. Psicol, 2018; 10(2):161-177.

7. DE-HITA-CANTALEJO C, et al. Accommodative and binocular disorders in preteens with computer vision syndrome: a crosssectional study. Annals of the New York Academy of Sciences, 2021; 1492(1): 73-81.

8. FARES J, et al. Musculoskeletal neck pain in children and adolescents: Risk factors and complications. Surgical neurology international, 2017; 8: 72.

9. HOKBY S, et al. Are Mental Health Effects of Internet Use Attributable to the Web-Based Content or Perceived Consequences of Usage? A Longitudinal Study of European Adolescents. JMIR mental health, 2016; 3(3): e31.

10. I OFFICE OF COMMUNICATIONS (OFCON). Children and parents: Media use and attitudes report 2018. Making Sense of Media, England, 2019. Disponível em: https://www.ofcom.org.uk/_data/assets/pdf_file/0024/134907/children-and-parentsmedia-use-and-attitudes-2018.pdf. Acesso em: 29 de julho de 2021.

11. KIM J, et al. Association between Exposure to Smartphones and Ocular Health in Adolescents. Ophthalmic epidemiology, 2016; 23(4): 269-276.

12. LISSAK G. Adverse physiological and psychological effects of screen time on children and adolescents: Literature review and case study. Environmental research, 2018; 164: 149-157.

13. MOHAN A, et al. Prevalence and risk factor assessment of digital eye strain among children using online e-learning during the COVID-19 pandemic: Digital eye strain among kids (DESK study-1). Indian journal of ophthalmology, 2021; 69(1): 140144.

14. MOON JH, et al. Association between video display terminal use and dry eye disease in school children. Journal of pediatric ophthalmology and strabismus, 2014; 51(2): 87-92.

15. MOON JH, et al. Smartphone use is a risk factor for pediatric dry eye disease according to region and age: a case control study. BMC ophthalmology, 2016; 16(1):188.

16. ORGANIZAÇÃO MUNDIAL DA SAÚDE (OMS). Relatório Mundial sobre a Visão. 2019. Disponível em: https://apps.who.int/iris/bitstream/handle/10665/328717/9789241516570-por.pdf. Acesso em: 28 de julho de 2021. RAMAMURTHY D, et al. A review of environmental risk factors for myopia during early life, childhood and adolescence. Clinical \& experimental optometry, 2015; 98(6): 497-506.

17. RECHICHI C, et al. Video Game Vision Syndrome: A New Clinical Picture in Children?. Journal of pediatric ophthalmology and strabismus, 2017; 54(6): 346-355.

18. REID CHASSIAKOS YL, et al. Council on communications and media. Children and Adolescents and Digital Media. Pediatrics, 2016; 138(5): e20162593.

19. ROSSETO JD, et al. Brazilian guidelines on the frequency of ophthalmic assessment and recommended exams in healthy children under 5 years of age. Arq Bras Oftalmol, 2020; 1: 1-10.

20. SAH RP, et al. Accommodative Behavior, Hyperopic Defocus, and Retinal Image Quality in Children Viewing Electronic Displays. Optometry and vision science: official publication of the American Academy of Optometry, 2020; 97(8): 628-640.

21. SHEPPARD AL, WOLFFSOHN JS. Digital eye strain: prevalence, measurement and amelioration. BMJ open ophthalmology, 2018; 3(1):e000146.

22. SOCIEDADE BRASILEIRA DE PEDIATRIA. Manual de Orientação Grupo de Trabalho Saúde na Era Digital (2019-2021). 2019. Disponível em: https://www.sbp.com.br/fileadmin/user_upload/_22246c-ManOrient_-_MenosTelas_MaisSaude.pdf. Acessado em: 28 de julho de 2021.

23. THEOPHANUS C, et al. Myopia prevalence and risk factors in children. Clinical ophthalmology, 2018; 12: 1581-1587.

24. $\mathrm{TOH} \mathrm{SH}$, et al. A prospective longitudinal study of mobile touch screen device use and musculoskeletal symptoms and visual health in adolescents. Applied ergonomics, 2020; 85: 103028. 